\title{
The STUN (STop UNhealthy) Alcohol Use Now trial: study protocol for an adaptive randomized trial on dissemination and implementation of screening and management of unhealthy alcohol use in primary care
}

Daniel E. Jonas ${ }^{1,2^{*}}$, Colleen Barclay ${ }^{1,2}$ (D) , Debbie Grammer ${ }^{3}$, Chris Weathington ${ }^{3}$, Sarah A. Birken ${ }^{4}$, Darren A. DeWalt ${ }^{2,5}$, Kimberly A. Shoenbill ${ }^{6,7}$, Marcella H. Boynton ${ }^{5}$, Monique Mackey ${ }^{3}$, Sean Riley ${ }^{1,2}$ and Samuel Cykert ${ }^{2,5}$

\begin{abstract}
Background: Unhealthy alcohol use is a leading cause of preventable deaths in the USA and is associated with many societal and health problems. Less than a third of people who visit primary care providers in the USA are asked about or ever discuss alcohol use with a health professional.

Methods/design: This study is an adaptive, randomized, controlled trial to evaluate the effect of primary care practice facilitation and telehealth services on evidence-based screening, counseling, and pharmacotherapy for unhealthy alcohol use in small-to-medium-sized primary care practices. Study participants will include primary care practices in North Carolina with 10 or fewer providers. All enrolled practices will receive a practice facilitation intervention that includes quality improvement (QI) coaching, electronic health record (EHR) support, training, and expert consultation. After 6 months, practices in the lower 50th percentile (based on performance) will be randomized to continued practice facilitation or provision of telehealth services plus ongoing facilitation for the next 6 months. Practices in the upper 50th percentile after the initial 6 months of intervention will continue to receive practice facilitation alone. The main outcome measures include the number (and \%) of patients in the target population who are screened for unhealthy alcohol use, screen positive, and receive brief counseling. Additional measures include the number (and \%) of patients who receive pharmacotherapy for AUD or are referred for AUD services. Sample size calculations determined that 35 practices are needed to detect a $10 \%$ increase in the main outcome (percent screened for unhealthy alcohol use) over 6 months.
\end{abstract}

\footnotetext{
*Correspondence: Daniel.Jonas@osumc.edu

'Division of General Internal Medicine and Geriatrics, Department of Internal

Medicine, The Ohio State University, 2050 Kenny Road, Columbus, Ohio 43221, USA

${ }^{2}$ Cecil G. Sheps Center for Health Services Research, CB 7590, University of North Carolina at Chapel Hill, Chapel Hill, NC 27599, USA

Full list of author information is available at the end of the article
}

C C The Author(s). 2021 Open Access This article is licensed under a Creative Commons Attribution 4.0 International License, which permits use, sharing, adaptation, distribution and reproduction in any medium or format, as long as you give appropriate credit to the original author(s) and the source, provide a link to the Creative Commons licence, and indicate if changes were made. The images or other third party material in this article are included in the article's Creative Commons licence, unless indicated otherwise in a credit line to the material. If material is not included in the article's Creative Commons licence and your intended use is not permitted by statutory regulation or exceeds the permitted use, you will need to obtain permission directly from the copyright holder. To view a copy of this licence, visit http://creativecommons.org/licenses/by/4.0/. The Creative Commons Public Domain Dedication waiver (http://creativecommons.org/publicdomain/zero/1.0/) applies to the data made available in this article, unless otherwise stated in a credit line to the data. 
Discussion: A successful intervention would significantly reduce morbidity among adults from unhealthy alcohol use by increasing counseling and other treatment opportunities. The study will produce important evidence about the effect of practice facilitation on uptake of evidence-based screening, counseling, and pharmacotherapy for unhealthy alcohol use when delivered on a large scale to small and medium-sized practices. It will also generate scientific knowledge about whether embedded telehealth services can improve the use of evidence-based screening and interventions for practices with slower uptake. The results of this rigorously conducted evaluation are expected to have a positive impact by accelerating the dissemination and implementation of evidence related to unhealthy alcohol use into primary care practices.

Trial registration: ClinicalTrials.gov NCT04317989. Registered on March 23, 2020.

Keywords: Alcohol, Unhealthy alcohol use, Screening, Counseling, Practice facilitation, Embedded telehealth, Primary care, Implementation science, Quality improvement

\section{Contributions to the literature}

Practice facilitation improves chronic disease care measures and adoption of evidence-based guidelines in primary care, but research is lacking on its effect on uptake of screening, counseling, and referral for unhealthy alcohol use.

Our study will evaluate whether practice facilitation can address and overcome key barriers to implementation (e.g., lack of a formal process) of these important services.

Our study will also evaluate the effect of embedded telehealth that connects patients with external counselors and services to overcome barriers to counseling in primary care, such as competing priorities, limited counseling experience and skills, and limited access to services.

\section{Background}

Unhealthy alcohol use is the third leading cause of preventable deaths among working-age adults in the USA and is associated with many societal and health problems [1-3]. It is a key contributor to recent declines in US life expectancy, especially among middle-aged, Americans and those living in rural areas [4,5]. National guidelines recommend no more than 4 drinks per day and 14 per week for men under 65 , and no more than 3 drinks per day and 7 per week for all women and for men 65 and older [6,7]. However, over $20 \%$ of primary care patients in the USA drink alcoholic beverages in excess of the recommended limits [8].

Multiple systematic reviews and recommendations have established that screening with brief validated questionnaires can accurately detect unhealthy alcohol use [9-11]. After detection, brief counseling interventions aim to reduce or eliminate risky drinking. Motivational interviewing techniques are commonly used in effective counseling interventions, and they can be an effective patient-centered approach for achieving behavior change $[12,13]$ Multiple systematic reviews have established the benefits of counseling (delivered by a variety of provider types) in primary care after screening, showing significant reductions in alcohol consumption [9-11]. For patients with alcohol use disorder (AUD), multiple more intensive treatment options are available; to date, no single intervention approach has been shown to be clearly superior to others in eliciting long-lasting reductions in unhealthy drinking. Twelve-step programs (e.g., alcoholics anonymous), cognitive behavior therapy, motivational enhancement therapy, and pharmacotherapy for AUD are among the commonly offered treatments [14].

Despite recommendations to screen for unhealthy alcohol use in the primary care setting, the burden of illness associated with it, and the existence of effective interventions, relatively few people who visit primary care providers in the USA are asked about alcohol use or ever discuss alcohol use with a health professional $[15,16]$. A major barrier is that many practices often lack a formal process for screening and delivery of appropriate interventions [17, 18]. Additional barriers include competing priorities, limited skills in the delivery of counseling in primary care, and limited access to services (e.g., counseling for AUD) [19-21]. More than half of US adults have visits with primary care providers [22], and these professionals enjoy extraordinarily high levels of trust from the public [23]. Primary care visits represent an important opportunity to address unhealthy alcohol use by providing a safe environment to discuss the issue with a trusted and skilled health professional.

A growing body of evidence indicates that practice facilitation, an implementation science method that uses a team-based, QI approach to guide organizational change, [24] is an effective strategy for implementing evidencebased practices. Practice facilitation interventions in healthcare involve helping practices with workflow analysis and redesign, EHR support including building templates and decision support tools, evidence-based protocols, population health lists, data feedback (e.g., run charts) and benchmarking, standing orders, and the 
utilization of the Model for Improvement, an integrated approach to process improvement that delivers quick and substantial results in quality and productivity in diverse settings [25]. A systematic review of 23 studies found that primary care practices that received practice facilitation were more likely to adopt evidence-based guidelines relative to those who did not receive practice facilitation (OR 2.76, 95\% CI, 2.18-3.43) [26]. A more recent 2018 systematic review of 25 randomized controlled trials (RCTs) and cohort studies found that practice facilitation improved a variety of chronic disease care measures, including those for cancer and cardiovascular disease [27]. None of the studies in either review focused on unhealthy alcohol use, indicating a gap in our knowledge on how to best help primary care practices address unhealthy alcohol use. Practice facilitation has the potential to address and overcome key barriers to implementation (e.g., lack of a formal process, limited counseling skills). However, some practices may lack the capacity to deliver counseling with their available resources and personnel. Such practices may benefit from having additional support through embedded telehealth services to connect their patients with external counselors and services, potentially overcoming barriers of competing demands and the lack of adequate skills to deliver effective counseling.

\section{Objectives and aims}

In October 2019, the U.S. Department of Health and Human Services, through the Agency for Healthcare Research and Quality (AHRQ), launched an initiative to fund six research studies intended to help hundreds of primary care practices screen for and reduce unhealthy alcohol use in primary care [28]. This protocol describes one of those studies. The STop UNhealthy (STUN) Alcohol Use Now study is an adaptive RCT that will be conducted in small and medium-sized primary care practices (10 providers or fewer) across North Carolina. The study aims to evaluate the effect of primary care practice facilitation on (1) uptake of evidence-based screening and brief intervention (SBI) for unhealthy alcohol use and (2) uptake of evidence-based counseling and pharmacotherapy for alcohol use disorder (AUD). For practices with slower uptake of SBI, the study will evaluate the effect of using telehealth services to deliver SBI for unhealthy alcohol use, and counseling and pharmacotherapy for AUD. Finally, the study will evaluate the effect of practice facilitation on the implementation of clinical practice and office systems changes to improve SBI and pharmacotherapy for AUD. Our primary hypotheses are that practice facilitation (and, in the case of slow-uptake practices, the further addition of telehealth) will improve processes of care for unhealthy alcohol use and increase rates of screening, identification, and appropriate interventions.

\section{Methods/design \\ Study setting}

North Carolina (NC) is the 10th largest state in the USA with a population of over 10 million and a racial and ethnic composition of 71\% White, 22\% Black, and 9.6\% Latino, respectively [29]. The burden of unhealthy alcohol use in NC remains large, accounting for an estimated 4000 deaths annually as well as substantial morbidity [30]. Approximately, $17 \%$ of adults in North Carolina engage in unhealthy alcohol use and 1 in 11 deaths among working-age adults (20-64 years old) in North Carolina are attributable to alcohol [31, 32]. The most common cause of alcohol-related deaths in NC is alcohol-impaired driving fatalities [33].

About 7400 physicians and 2000 advanced practice providers work mainly as primary care providers in North Carolina, according to 2019 NC licensure data in the North Carolina Health Professions Data System [34]. Practice size estimates vary according to practice definition (tax ID\# vs. single geographic location), but based on the provider to practice ratios, we estimate that there are approximately 2200 primary care practices in NC. Thirty to $40 \%$ of these remain unaffiliated with larger health systems, and almost all have fully implemented EHRs. The practices eligible for enrollment in STUN are skewed toward rural areas.

\section{Research design}

STUN Alcohol Use Now is an adaptive, randomized, controlled trial to evaluate the effect of primary care practice facilitation and the effect of using telehealth services on evidence-based screening, counseling, and pharmacotherapy for AUD (Fig. 1).

The design is considered an adaptive trial design because inclusion in the randomized portion of the study is based on practice performance during the initial 6 months of the intervention. All enrolled practices will receive the practice facilitation intervention. We followed the SPIRIT guidance for reporting the content of this study protocol and completed the SPIRIT checklist (supplemental file) and the SPIRIT figure (Fig. 2) [35].

After practices have received the practice facilitation intervention for 6 months, those in the lower 50th percentile of performance will be block randomized to continued practice facilitation or to using telehealth services plus continued practice facilitation for the next 6 months. The random allocation sequence will be generated using Microsoft excel by a data analyst who is not involved in recruitment or practice facilitation. The random allocation sequence will be stored in a password- 


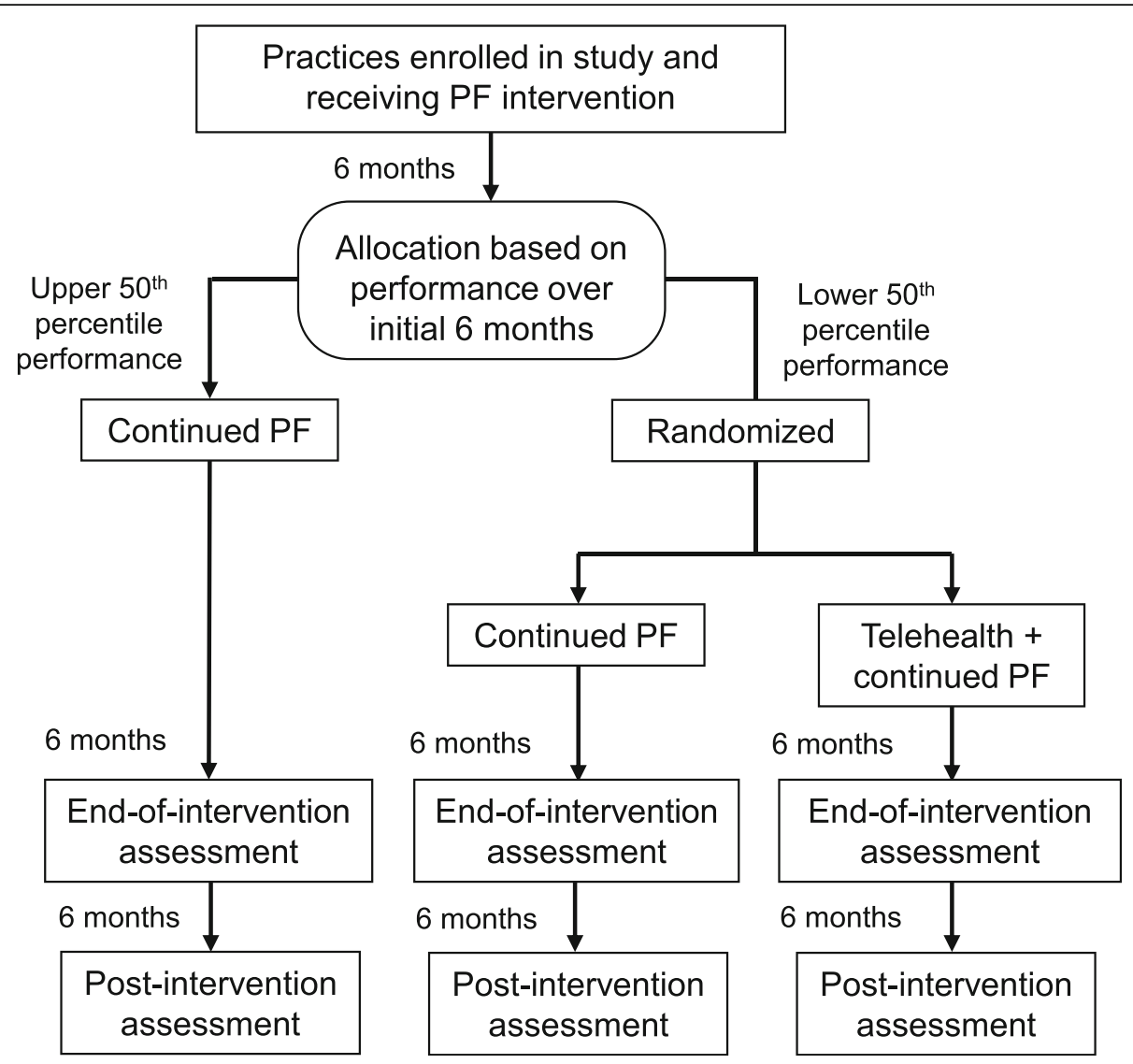

Fig. 1 Study flow diagram

protected electronic file and concealed from all other team members involved in recruitment and practice facilitation until after a practice has been determined to be in the lower 50th percentile of performance and has received the practice facilitation intervention for 6 months. The performance assessment over the initial 6 months will be based on the percentage of patients screened for unhealthy alcohol use and the percentage of patients who receive brief counseling when it is indicated (after screening indicates unhealthy alcohol use). Randomizing the lower 50th percentile will allow us to assess (1) whether the provision of telehealth services accelerates uptake for practices with slower uptake and (2) whether "staying the course" with ongoing practice facilitation is an effective strategy for those with slower uptake (comparing whether they catch up to the upper 50th percentile). Practices in the upper 50th percentile over the initial 6 months will continue to receive the practice facilitation intervention for another 6 months.

\section{Practice recruitment}

Practice recruitment will be conducted by the practice facilitators (i.e., practice coaches). Due to COVID-19related constraints, recruitment will be largely conducted on a virtual basis using phone calls, emails, and video conferencing. For the purpose of planning, guiding recruitment activities, and refining recruitment strategies, the research team will create and provide informational materials, schedule informational webinars with potential practice representatives, and participate in regular educational sessions with the practice facilitators. Based on enrollment targets set by the funder, hoping to reach and help as many practices as might be feasible, we initially aimed to enroll up to 135 small to medium-sized primary care practices. Our sample size calculations indicated that many fewer practices are needed to assess the effect of the intervention on our main outcomes. Specifically, 35 practices would be needed to detect a $10 \%$ increase in screening for Aim 1, and fewer practices would be needed if the magnitude of the increase in screening after the intervention is larger than 10\% (as detailed in the Statistical Power section under Aim 1). Competing demands and constraints due to the pandemic tempered enrollment, leading the research team to adjust the enrollment targets to those based on our sample size calculations. Practices are eligible for enrollment if 10 or fewer providers occupy a single location and do not receive facilitation services specifically related 


\begin{tabular}{|c|c|c|c|c|c|c|c|}
\hline \multirow[b]{3}{*}{ TIMEPOINT } & \multicolumn{7}{|c|}{ STUDY PERIOD } \\
\hline & \multirow{2}{*}{$\begin{array}{l}\text { Enrolment } \\
-3 \text { months }\end{array}$} & \multicolumn{2}{|c|}{$\begin{array}{l}\text { Practice Facilitation } \\
\text { Intervention }\end{array}$} & \multirow[t]{2}{*}{$\begin{array}{c}\text { Randomiz } \\
\text { ation }\end{array}$} & \multicolumn{2}{|c|}{$\begin{array}{l}\text { Telehealth and } \\
\text { Continued Practice } \\
\text { Facilitation } \\
\text { Intervention }\end{array}$} & \multirow{2}{*}{$\begin{array}{c}\begin{array}{c}\text { Close- } \\
\text { out }\end{array} \\
\begin{array}{c}18 \\
\text { months }\end{array}\end{array}$} \\
\hline & & 0 & $\begin{array}{c}6 \\
\text { months }\end{array}$ & & $\begin{array}{c}6 \\
\text { months }\end{array}$ & $\begin{array}{c}12 \\
\text { months }\end{array}$ & \\
\hline \multicolumn{8}{|l|}{ ENROLMENT: } \\
\hline \multirow{2}{*}{$\begin{array}{l}\text { Eligibility screen } \\
\text { Informed consent }\end{array}$} & $X$ & & & & & & \\
\hline & $\mathrm{X}$ & & & & & & \\
\hline Allocation & & & & $\mathrm{X}$ & & & \\
\hline \multicolumn{8}{|l|}{ INTERVENTIONS: } \\
\hline \multicolumn{8}{|l|}{ Practice Facilitation } \\
\hline \multicolumn{8}{|l|}{$\begin{array}{r}\text { Continued Practice } \\
\text { Facilitation }\end{array}$} \\
\hline \multicolumn{8}{|l|}{$\begin{array}{r}\text { Telehealth + } \\
\text { Continued Practice } \\
\text { Facilitation }\end{array}$} \\
\hline \multicolumn{8}{|l|}{ ASSESSMENTS: } \\
\hline $\begin{array}{r}\text { Implementation } \\
\text { Support }\end{array}$ & & & $\mathrm{X}$ & & $X$ & & \\
\hline \multirow{3}{*}{$\begin{array}{r}\text { Practice capacity for } \\
Q I \\
\text { Organizational } \\
\text { Readiness } \\
\text { Implementation } \\
\text { Policies and } \\
\text { Practices }\end{array}$} & & $x$ & & & & $x$ & $X$ \\
\hline & & $\mathrm{X}$ & & & & & \\
\hline & & & $\mathrm{X}$ & & $\mathrm{X}$ & & \\
\hline $\begin{array}{r}\text { Implementation } \\
\text { Climate }\end{array}$ & & & & & & $x$ & $X$ \\
\hline $\begin{array}{r}\text { Implementation } \\
\text { Effectiveness }\end{array}$ & & $\mathrm{X}$ & $\mathrm{X}$ & & $\mathrm{X}$ & $x$ & $\mathrm{X}$ \\
\hline Contextual Factors & & $\mathrm{X}$ & $\mathrm{X}$ & & $x$ & $x$ & $\mathrm{x}$ \\
\hline $\begin{array}{r}\text { Telehealth } \\
\text { Acceptability }\end{array}$ & & & & & & $\mathrm{x}$ & $\mathrm{X}$ \\
\hline
\end{tabular}

Fig. 2 SPIRIT figure: Schedule of enrolment, interventions, and assessments

to unhealthy alcohol use. Enrolling practices agree to the following: (1) work with practice facilitators for 4 to $8 \mathrm{~h}$ a month to implement an evidence-based screening process as well as a process for counseling and/or referring patients with unhealthy alcohol use, (2) participate in webinars conducted by project personnel about the screening and brief counseling process as well as how and when to prescribe medications for AUD, (3) respond to surveys about the practice environment and the improvement process, and (4) collect implementation effectiveness data on a monthly basis with help from practice facilitators.

Potential barriers to practice recruitment include fear of financial risk, dedicating practice resources to other new activities such as Medicaid reform or Accountable Care Organization participation to the exclusion of participation in this study, as well as concerns related to staffing issues, competing priorities, and lack of time. These barriers have been compounded during the
COVID-19 pandemic as practices struggle to meet the demands of caring for patients in a changed environment and contend with new financial pressures arising from reduced patient visits. Practice facilitation will attempt to mitigate these barriers by helping practices adapt-for example, by streamlining workflows. During recruitment, we will also emphasize current evidence that the increase in social isolation, household pressures, and economic stress during the pandemic has been associated with an increase in unhealthy alcohol use [36-38].

\section{Practice support intervention}

The NC Area Health Education Centers (AHEC) program has the permanent statewide infrastructure and highly trained personnel to support and deliver practice facilitation services and has developed strong relationships with primary care practices [39]. Participating practices will receive direct practice facilitation over the 12-month intervention period from NC AHEC 
personnel. The facilitator will ensure that key drivers for improvement are identified and that the practice is comfortable with implementing the improvement with rapidcycle tests of change. Practices will receive $1-2 \mathrm{~h}$ of direct practice facilitation services per month and be expected to apply tests of change using a Plan-Do-StudyAct (PDSA) approach [40] fairly independently, confirmed and coached by a member of the facilitation team. The facilitator will ensure that the practice has established specific workflows for the unhealthy alcohol use measures and conduct periodic data checks to ascertain progress, sharing the results of these data checks with the practice (in an audit and feedback fashion). Webinars and video recordings will serve as additional tools that facilitators can use to educate practices on best practices for alcohol screening, counseling, and interventions. Expert consultation with physician faculty will be available, primarily virtually, to supplement facilitation efforts. While practice facilitation was initially planned as a combination of face-to-face meetings, phone calls, web-based video meetings, and email communication, remote communications will be the primary communication modality until risks from the pandemic have subsided. Facilitation meetings will emphasize the implementation of evidence-based protocols and the use of clinical algorithms by the following:

1. Forming clinical QI teams to engage the practice (or its participating clinicians) in a high standard of care delivery, including the use of standing orders, EHR templates, and clinical decision support tools.

2. Establishing human workflows including teambased roles to use these practice tools

3. Optimizing the use of the EHR to perform monthly data pulls to guide and evaluate the progress of the screening process, counseling, pharmacologic treatment, and associated referrals.

4. Developing spreadsheet registries and other electronic tools for practices lacking the EHR capabilities to develop the data resources described above.

5. Assisting the practices in optimizing billing for reimbursable SBI services.

6. Working with practices to develop proactive assigned roles and responsibilities to prepare the clinical team to develop needed care and engage patients.

7. Providing lists of available counseling and referral resources by region and county, to be used when primary care clinicians encounter patients whose unhealthy alcohol use, AUD, or comorbid behavior health conditions exceed the clinician's comfort level or expertise.
8. For practices randomized to the telehealth group, protocols for scheduling and utilizing telehealth services will be developed.

The practice facilitator workforce is shovel-ready, with facilitators trained in 49 coaching competencies and training content continually updated to keep facilitators prepared to help practices respond to emergent needs in the field. They have prior experience working with practices on multiple topics. Upon enrollment, the facilitator establishes an intervention start date with the practice, taking into consideration current and upcoming conditions and priorities at the practice. Early tasks include assessing baseline alcohol screening rates and determining EHR reporting capabilities. The 12-month intervention period begins when the facilitator begins to work regularly with the practice on the processes described above.

\section{Evaluation framework}

Implementation of practice facilitation to support the uptake of an evidence-based screening, brief counseling intervention, and referral to alcohol treatment represents a major innovation for addressing the substantial health burden of unhealthy alcohol use. Briefly, effective implementation is a function of the implementation support the practice receives and the policies and practices it employs to support innovation use.

Organized QI effort and capability will be the key driver of improvement, creating an environment in which primary care practices can embrace the implementation of the Chronic Care Model [41]). The Chronic Care Model emphasizes that practices use clinical decision support, clinical information systems, optimal delivery system design, self-management support, and community linkages to create prepared and proactive care teams and informed and motivated patients, leading to improved health outcomes [42]. The elements of the model fit well with the implementation of SBI for unhealthy alcohol use and pharmacotherapy for AUD.

Our primary hypotheses, that practice facilitation (and, in the case of slow-uptake practices, the further addition of telehealth), will improve processes of care for unhealthy alcohol use are motivated by adult learning theory and social cognitive theory (SCT) [43-45]. Adult learning theory posits that people prefer to learn based on real-life problems, by setting realistic goals, listening to their peers, and experiencing success when they experiment with improvement efforts. STUN Alcohol Use Now's practice facilitation adheres to the preferences of the targeted adult learners (clinicians and practice staff) in primary care practices (Table 1). 
Table 1 STUN Alcohol Use Now's hypothesized influence

\begin{tabular}{ll}
\hline Adult learners learn... & STUN Alcohol Use Now approach \\
\hline Based on real-life problems & Practice facilitation, expert consultation, and training modules will incorporate real problems \\
By setting realistic goals & Practice facilitation, expert consultation, and training modules will emphasize realistic goals \\
By listening to their peers & Practice facilitation, expert consultation, and training modules will incorporate peers in their stories \\
By experiencing success & Use of EHR reports, run charts, and positive reinforcement by facilitators \\
\hline
\end{tabular}

\section{Data and safety monitoring}

Our Data Safety and Monitoring Plan (DSMP), commensurate with the low degree of risk involved in participation, will focus on monitoring and minimizing risks to participating practices, careful monitoring of the study's progress, protecting the confidential medical and personal information of subjects, and ensuring the validity and integrity of the collected data.

The principal investigator will be responsible for carrying out the DSMP. Key personnel involved with the logistics of practice enrollment, participation, and followup (e.g., the overall project manager, AHEC project manager, and data manager) will meet regularly (at least quarterly) to review, among other things, progress on accrual, implementation, data collection, and adverse events. Many of these individuals, along with the study investigators, will meet weekly to address ongoing study issues including, among other things, progress on accrual (practice recruitment and retention), follow-up, and adverse events. The project manager will then prepare the needed reports (Table 2) for the PI.

Annually, the PI will prepare a written report addressing the study's progress and safety, which will be provided to the IRB as part of the annual renewal submission.
AHEC technical support personnel will assist participating practices with producing the discrete EHR data fields necessary to accrue the measures and reliably transfer them for processing. The practice facilitators will work with practice teams to create workflows that assure data entry into appropriate fields. Secure data will be uploaded to the project database on a monthly basis via an online system we can monitor for timeliness and completeness of data entry. The regular data monitoring during this study will allow us to identify missing data and, when feasible, obtain this information through phone calls or email follow-up. Where EHRs of small practices have limited capability to produce reports, we will provide web-based data entry tools and registries instead. The PFs will monitor, remind, and encourage the practices to complete surveys.

Project data is housed at the parent research center's dedicated research computing servers for the analysis of large-scale research datasets. Access to these servers requires two-factor authentication, a restricted VPN connection, and is limited to specific programmers authorized by the center's senior management. Once connected to the system, each programmer can access only data needed for their specific project, by way of operating-system-level security groups. In addition to

Table 2 Data and Safety Monitoring Plan

Data type and description
Practice recruitment and retention
Study performance (via Practice Facilitator contact logs, we will capture
number and types of personnel working with practices to support
implementation, number, and type of interactions between project staff/
consultants and practices, number of practices reached by the
implementation, number of clinicians engaged, and percentage of PF
contacts with a practice that are in-person)
Implementation effectiveness (assessed via required measures.)

Risks to participating practices (practice facilitators will be trained to monitor the time commitment of the practice to the project and to note any disruption in practice workflow or functions)

Adverse events (AEs), such as breach of confidentiality

Stopping rules regarding benefits and harms

Stopping rules regarding statistical power

\section{Frequency of review}

Weekly, with monthly summaries for PI including a graphic of projected vs. actual

Monthly summary to the PI

All significant protocol deviations will be reported to the IRB during the annual review process

A monthly summary, including run charts, will be prepared from uploaded encrypted and secure data submitted from the practices to our database

Monthly summary to the PI

Practice facilitators will be trained to identify and report AEs as they occur to the AHEC project manager and overall project manager. The PI will be responsible for reporting AEs, as they occur, to the IRB, using UNC IRB definitions, standards, and forms

Not applicable to the study

Not applicable to the study 
these programmers, data is accessed by study personnel who have completed all required security and privacy training.

To minimize risks, all efforts will be made to collect data in a manner that protects a person's right to confidential participation. Survey links that are sent, after the consent, to practice staff via email will remind staff that they may be completed at a time and place of their own choice. They also will be offered the option to complete the questions by phone if more comfortable for them. Programmers and research staff who work with sensitive data are required to complete appropriate HIPAA training with periodic updates, complete and comply with all institution training, Security Policies, and provisions.

\section{Constructs and measures}

\section{Implementation support}

Using practice facilitation contact logs, we will capture the number and type of interactions between project staff/consultants and practices, the number of practices reached by the implementation, and the number of clinicians engaged.

\section{Practice capacity for QI}

Practice capacity for QI encompasses 2 constructs, change process capability and adaptive reserve. We will assess the former using the 32-item Change Process Capacity Questionnaire [46] and the latter using the 3item adaptive reserve (i.e., capacity for change) scale [47, 48]. Both the CPCQ and the adaptive reserve scale exhibit reliability and known-group validity $[46,49]$.

\section{Organizational readiness for change}

Organizational readiness for change refers to the extent to which organizational members are psychologically and behaviorally prepared to implement organizational change [50-52]. We will measure readiness using the 12-item Organizational Readiness for Implementing Change (ORIC) scale [50] ORIC has demonstrated reliability, content validity, structural validity, structural invariance, and known-groups validity [50].

\section{Implementation policies and practices}

Implementation policies and practices are the strategies that an organization puts into place to support innovation use [53, 54]. We will measure these with the Key Driver Implementation Scale (KDIS), which uses a 5-point, behaviorally anchored, ordinal scale that covers multiple fundamental drivers of improvement.

\section{Implementation climate}

Implementation climate refers to organizational members' "shared summary perception of the extent to which their use of a specific innovation is rewarded, supported, and expected within their organization" [55]. We will measure implementation climate using a 6-item scale with demonstrated reliability, structural validity, structural invariance, known-groups validity, and predictive validity.

\section{Implementation effectiveness}

Implementation effectiveness refers to the consistency and quality of innovation use [53]. Implementation effectiveness is conceived here as an organization-level construct describing organizational members' pooled consistency and quality of innovation use (i.e., evidencebased screening, counseling, referrals, and pharmacotherapy) [53, 56, 57]. We will assess implementation effectiveness using the measures in Table 3.

\section{Telehealth acceptability}

Telehealth acceptability for those practices provided with telehealth services will be measured using a brief survey based on the Technology Acceptance Model [58, 59].

\section{Data collection}

Table 4 outlines data collection, measures, sources, and timing for each construct.

\section{Practice facilitator contact logs}

To capture implementation support, practice facilitators will record the date, duration, and mode of each practice contact. We will use these data to measure the level of implementation support provided to each practice.

\section{Provider/staff survey}

We will survey providers and staff to assess practice capacity for QI at baseline, end-of-intervention (12 months after baseline), and 6 months post-intervention (18 months after baseline); organizational readiness to implement change at baseline; and implementation climate at end-of-intervention and 6 months post-intervention. To document contextual factors, surveys will also capture relevant practice characteristics, general patient population and demographics, and EHR capabilities. The survey sample at each time point will consist of 3-5 providers and staff members depending on practice size.

\section{Practice facilitator ratings of practices' progress}

Practice facilitators will assess practices' progress in implementing clinical practice and organizational changes to support improvement in screening, brief intervention, and referral to treatment (SBIRT) (implementation policies and practices) at monthly intervals. Ratings will focus on the extent to which practices have implemented multiple key drivers of improved SBIRT provision and level of leadership and team engagement. 
Table 3 Implementation effectiveness measures

\begin{tabular}{|c|c|}
\hline Measure & Description \\
\hline \# and \% of patients screened & $\begin{array}{l}\text { Number and percentage of adult patients with documentation of screening for unhealthy alcohol use } \\
\text { with the screening questions recommended by NIAAA }\end{array}$ \\
\hline$\#$ and $\%$ of patients with a positive screen & Number and percentage of adult patients with a positive initial screen \\
\hline $\begin{array}{l}\text { \# and } \% \text { of patients completing the } \\
\text { AUDIT }\end{array}$ & $\begin{array}{l}\text { Number and percentage of those with a positive initial screen }{ }^{a} \text { who go on to complete the } 10 \text {-question } \\
\text { AUDIT (the next step in assessment after an initial positive screen) }\end{array}$ \\
\hline $\begin{array}{l}\text { \# and } \% \text { of patients who receive brief } \\
\text { counseling }\end{array}$ & $\begin{array}{l}\text { Number and percentage of adult patients with documentation of brief counseling for risky drinking, } \\
\text { when indicated/appropriate }\end{array}$ \\
\hline \# and \% of patients who have AUD & Number and percentage of adult patients with documented ICD diagnoses of AUD \\
\hline $\begin{array}{l}\text { \# and \% of patients who receive } \\
\text { pharmacotherapy for AUD }\end{array}$ & $\begin{array}{l}\text { Number and percentage of adult patients with AUD who receive evidence-based pharmacotherapy with } \\
\text { naltrexone, acamprosate, disulfiram, or topiramate }{ }^{b}\end{array}$ \\
\hline $\begin{array}{l}\# \text { and } \% \text { of patients referred to specialty } \\
\text { care for AUD }\end{array}$ & $\begin{array}{l}\text { Number and percentage of adult patients with AUD referred to specialty care (e.g., psychiatry, CBT, } \\
\text { motivational enhancement therapy, } 12 \text {-step programs) }\end{array}$ \\
\hline
\end{tabular}

AUD alcohol use disorder, AUDIT alcohol use disorders identification test, CBT cognitive behavioral therapy, ICD International Classification of Diseases, NIAAA National Institute on Alcohol Abuse and Alcoholism

aDo you sometimes drink beer, wine, or other alcoholic beverages? (If yes) How many times in the past year have you had 5 or more (for men 64 and younger)/4 or more (for women of any age, and men 65 and older) drinks in a day? [6] Response of 1 or more is a positive initial screen

${ }^{\mathrm{b}}$ Topiramate is not FDA-approved for AUD, but it has been shown to be beneficial (e.g., for reducing heavy drinking days) [14]

\section{Chart review}

To assess screening rates at baseline, practice facilitators will assist practice staff in querying their EHR or conducting a chart review to determine the number and proportion of patients who have had evidence-based screening over the previous 2 years.

\section{EHR/informatics}

Practices will obtain performance data on the implementation effectiveness (uptake) from their EHRs or by recording the data in a registry, the creation of which the practice facilitators and project team will guide. These data will be entered as aggregate counts and percentages for each practice (no protected health information [PHI] will be included) in a dedicated, online tool that feeds into the project database. The implementation effectiveness measures will be collected at baseline, during the intervention, at the end of the intervention, and post-intervention (6 month post-intervention follow-up, i.e., 18 months after baseline).

\section{Data analysis}

Aim 1: Evaluate the effect of primary care practice facilitation on uptake of evidence-based SBI

Our primary hypotheses are that practice facilitation will increase the number and percentage of patients in a practice who are: (a) screened for unhealthy alcohol use, (b) identified to have unhealthy alcohol use, and (c)

Table 4 Theoretical constructs, measures, data sources, and data collection timing

\begin{tabular}{|c|c|c|c|}
\hline Construct & Measure & Source & Timing \\
\hline $\begin{array}{l}\text { Implementation } \\
\text { support }\end{array}$ & Frequency, duration, mode, and purpose of practice contacts & PF contact logs & I \\
\hline $\begin{array}{l}\text { Practice capacity for } \\
\text { Ql }\end{array}$ & $\begin{array}{l}\text { Change Process Capacity Questionnaire (CPCQ) } \\
\text { Adaptive Reserve Questionnaire }\end{array}$ & Provider/staff survey & $B, E, F$ \\
\hline $\begin{array}{l}\text { Organizational } \\
\text { readiness }\end{array}$ & Organizational Readiness for Change Questionnaire (ORIC) & Provider/staff survey & B \\
\hline $\begin{array}{l}\text { Implementation } \\
\text { policies and practices }\end{array}$ & $\begin{array}{l}\text { Key Driver Implementation Scale (KDIS) and type and quantity of strategies } \\
\text { implemented }\end{array}$ & PF ratings & 1 \\
\hline $\begin{array}{l}\text { Implementation } \\
\text { climate }\end{array}$ & Implementation Climate Questionnaire & Provider/staff survey & $E, F$ \\
\hline $\begin{array}{l}\text { Implementation } \\
\text { effectiveness }\end{array}$ & $\begin{array}{l}\text { Number and \% of patients in the target population who are screened for unhealthy } \\
\text { alcohol use, screen positive, receive brief counseling, have AUD, receive } \\
\text { pharmacotherapy, referred for AUD }\end{array}$ & $\begin{array}{l}\text { Data forms on the website, } \\
\text { chart review, or direct from } \\
\text { EHR }\end{array}$ & $\begin{array}{l}B, I, E \\
F\end{array}$ \\
\hline Contextual factors & Practice characteristics, patient population, EHR capabilities & Provider/staff survey & $\begin{array}{l}B, I, E, \\
F\end{array}$ \\
\hline $\begin{array}{l}\text { Telehealth } \\
\text { acceptability }\end{array}$ & Acceptability to practice; satisfaction with workflow and quality & Provider/staff survey & $\mathrm{E}, \mathrm{F}$ \\
\hline
\end{tabular}

$\overline{P F}$ practice facilitator, QI quality improvement, EHR electronic health record, $A U D$ alcohol use disorder, Timing: $B$ baseline, $I$ intervention (weekly/monthly), $E$ end of the intervention, $F$ 6-month post-intervention (i.e., 18 months after baseline) 
provided with brief counseling. Our secondary hypothesis is that practice capacity for QI, organizational readiness to implement change, implementation climate, and contextual factors will moderate the effect of primary care practice facilitation on the use of evidence-based SBI for unhealthy alcohol use.

Statistical analysis For each practice, percent screened for unhealthy alcohol use will be computed using all data available from the baseline (henceforth "baseline") as well as in each of the two quarters up to 6 months. 95\% confidence intervals will be calculated with the percentages compared using repeated measures ANOVA for an overall test of change following practice facilitation, as well as for differences in percentages for each of the post-facilitation quarters relative to pre-baseline. The changes in percent screened will be evaluated with paired $t$ tests looking at differences between each pair of quarters at alpha $=.05$. The primary analysis will be based on a comparison of the baseline versus the 2 nd quarter following practice facilitation, where the impact of practice facilitation is likely to be greatest. The repeated measures ANOVA will allow us to potentially detect a delay in effectiveness. Similar analyses will be conducted using percent identified with unhealthy alcohol use and for the percent provided with counseling, based on the data from baseline and up to 6 months after practice facilitation initiation. The analyses will be redone adjusting for the calendar time period to control for potential changes occurring naturally over time. As a secondary analysis, we will examine proportion trajectories using a mixed modeling approach, iteratively testing linear, quadratic, and cubic time functions (Level 1); interacting those time functions with intervention conditions (Level 2); and controlling for key covariates (e.g., clinic size). Relative model fit statistics (e.g., Akaike information criterion $[\mathrm{AIC}]$ ) will be used to inform the selection of the final model.

We will examine the effects of practice characteristics, such as organizational readiness and implementation environment on the impact of practice facilitation. Descriptive statistics will be calculated for practice-level variables. Linear regression models will be fit to practice level change scores based on differences in the two quarters after practice facilitation compared to the baseline percentage. Generalized estimating equations will be used to fit models simultaneously using the two change scores. Each moderating variable will be screened in univariate models, with significant variables entered in a multivariable model. AIC will be used to choose the final model.

Secondary analyses will consider quarterly data collected after 6 months to the end of follow-up at 12 months, and to the post-intervention follow-up, to evaluate the effect of practice facilitation as well as the effect of telehealth combined with practice facilitation. This will involve analyses similar to those above in which all practices that are randomized to no telehealth are grouped together, while higher-performing practices at 6 months randomized to telehealth (but not receiving telehealth) and lower performing practices randomized to telehealth constitute a second group. Change scores will be calculated from baseline. Average percent improvements from baseline between the two groups will be compared at each quarter after 6 months using twosample $t$ tests, with an overall test based on repeated measures ANOVA. Adjustment for practice-level characteristics will be explored using GEE. Survey data collected from multiple clinic staff/health care providers will be analyzed using a mixed modeling approach, with a person (Level 1) nested within the clinic (Level 2) where appropriate, three-level models with repeated measures (Level 1), within person (Level 2), and within clinic (Level 3) will be employed when considering time effects for these data.

Patterns of missing data will be assessed descriptively. Associations between practice level covariates and missingness will be explored, with differences formally tested using logistic regression models for binary missingness variables. Inverse weighting based on missingness probabilities will be employed to adjust for the effects of practice level covariates on missingness, where appropriate. Primary analyses will follow the intention to treat principles.

The main outcomes are being measured at the practice level (e.g., did the practice increase their rates of screening for unhealthy alcohol use), not at the individual patient level, and the unit of inference is the practice (not the individual patient). Therefore, the trial was not designed as a cluster trial and there is not a need to account for clustering; trials focused on outcomes measured at the group/practice level can be regarded as standard clinical trials with respect to the estimation of sample size and analysis approach [60, 61].

Statistical power We estimate that a very low percentage $(<5 \%)$ currently receives recommended screening. On average, based on our experience and prior evaluations we expect about $45 \%$ of patients within each practice to receive screening following practice facilitation, by 6 months. For unhealthy alcohol use, preliminary data suggest that about $25 \%$ of those screened will have unhealthy alcohol use identified (roughly 7.5\% of all adults served by the practice). Conservatively, if there is a $10 \%$ increase in the percentage (of either screening or detection of unhealthy alcohol use from baseline to 6 months) on average across practices, with a standard deviation in the percentage improvement of $10 \%$, then a one-sample 
$t$ test at level 0.05 has at least $80 \%$ power to detect that improvement with sample size 34 . We expect that the increase in the percentage of adults screened would be higher than $10 \%$ and that fewer practices would be needed to detect a significant increase; our prior work (when our intervention was less developed) showed an increase of more than $30 \%$ over 6 months [19-21]. Thus, the proposed design has very good power to detect realistic effect sizes for improved screening and detection of unhealthy alcohol use, even with considerable dropout and/or missing data (even more than 30\% missingness).

\section{Aim 2: Evaluate the effect of practice facilitation on uptake of evidence-based counseling and pharmacotherapy for AUD}

Our hypotheses are that practice facilitation will increase the number and percentage of patients in a practice who are (a) identified to have AUD, (b) provided with pharmacotherapy for AUD, and (c) referred to specialty care for AUD.

Statistical analysis As in Aim 1, for each practice, the percent identified with AUD will be computed using all data available. 95\% confidence intervals will be calculated with the percentages compared using repeated measures ANOVA for an overall test of change following practice facilitation, with paired $t$ tests looking at differences between each pair of quarters. The primary analysis will be based on a comparison of the baseline and the 2nd quarter following practice facilitation. Similar analyses will be conducted using percent-provided pharmacotherapy and referred to specialty care for AUD, based on the data from baseline and up to 6 months after practice facilitation initiation. Additional secondary analyses will consider quarterly data collected after 6 months from baseline to the end of follow-up at 12 months, and to the post-intervention follow-up (18 months), using the intent to treat framework described for Aim 1. Missingness for Aim 2 endpoints will be assessed as in Aim 1. Mixed modeling will be used to compute trajectory models as described for Aim 1.

Statistical power Detection of previously unknown AUD via screening in primary care is relatively rare, on the order of $1 \%$ among adults with no history of AUD. Assuming $45 \%$ of adults are screened over 6 months, we would expect an increase of roughly $0.45 \%$ from baseline following practice facilitation. Among those with AUD, we anticipate a relatively large increase in pharmacotherapy for AUD, from 0 to $33 \%$ on average. To detect an increase of $0.45 \%$ in AUD detection on average with a standard deviation of $1 \%$ using a one-sample $t$ test at level 0.05 , sample sizes of 39 and 52 give $80 \%$ and $90 \%$ power to detect such differences. The increase in pharmacotherapy for AUD for those identified as having AUD is quite large and the one-sample $t$ tests have very large power to detect such improvements.

\section{Aim 3: For practices with slower uptake, evaluate the effect of telehealth services on the use of evidence-based (a) SBI for unhealthy alcohol use and (b) counseling and pharmacotherapy for AUD}

Our primary hypotheses are that, compared with continued practice facilitation, practices randomized to the provision of telehealth services will increase the number and percentage of patients who are (a) provided with brief counseling for unhealthy alcohol use, (b) provided with pharmacotherapy for AUD, and (c) referred to specialty care for AUD. Our secondary hypothesis is that telehealth services for counseling will be acceptable to small to medium-size primary care practices.

Statistical analysis Practices in the lower 50th percentile at 6 months will be randomized to telehealth or not. The primary analysis will be an ITT analysis based on a comparison of changes in counseling, pharmacotherapy, and specialty care from 6 months to 12 months (end-ofintervention). Such changes will be calculated based on a comparison of quarterly percentages, where 3-6 months serves as a baseline and 6-9 and 9-12 months as the post-randomization quarters. Note that for this aim, the 6-month baseline differs from the baseline in Aims 1 and 2. Average percent changes and $95 \%$ confidence intervals will be computed for SBI and pharmacotherapy in the two intervention arms for 6-9 and 9-12 months. The two arms will be compared using repeated measures ANOVA and two-sample $t$ tests at each quarter at level .05. The main focus is on the changes from 3-6 months to 9-12 months, where the impact of telehealth is likely to be greatest. The repeated measures ANOVA will allow us to potentially detect increased impact over time. Secondary analyses will assess the post-intervention follow-up (focusing on change from 3-6 to 15-18 months) and acceptability of telehealth. Acceptability percentage will be calculated across practices, along with 95\% confidence intervals. Mixed modeling will be used to assess the impact of practice-level characteristics on acceptability on the part of staff/healthcare providers.

Statistical power The focus is on detecting differences in the changes from 6 to 12 months for practice facilitation + telehealth versus practice facilitation without telehealth. Differences will be tested at level .05 using twosample $t$ tests based on an ITT analysis of percentage changes in the two randomized groups. Assuming that the standard deviation of the percent changes is .15 in each group (and a between-group difference of 20\%), a sample size of 9 per group gives $80 \%$ power and a 
sample size of 12 gives $90 \%$ power. The resulting effect sizes are moderate in size and consistent with previous results.

\section{Aim 4: Evaluate the effect of practice facilitation on the implementation of clinical practice and office systems changes to improve evidence-based SBI and pharmacotherapy}

Our primary hypothesis is that practice facilitation will increase the implementation of clinical practice and office systems changes to improve evidence-based SBI and pharmacotherapy. Our secondary hypotheses are that (a) practice capacity for QI, organizational readiness to implement change, and contextual factors will moderate the effect of practice facilitation on the implementation of clinical practice and office systems changes and (b) provision of telehealth services will increase implementation of clinical practice and office systems changes among practices with slower uptake.

Statistical analysis This aim is structured to explore the impact of practice facilitation and telehealth on the implementation of clinical practice and office systems changes. The analysis plan mirrors that in Aims 1 and 2 . The first set of analyses is based on outcomes from the first two quarters from baseline to 6 months in which the effect of practice facilitation is explored, independently of telehealth. The second set of analyses compares the effect of practice facilitation with and without telehealth using quarterly data from 6 to 12 months. Moderating effects of practice-level characteristics will be explored in both sets of analyses.

Statistical power These power calculations are similar to those for Aims 1 and 2 which focused on the impact of practice facilitation. Here, the primary endpoint is detecting a difference in KDIS score from baseline, which is evaluated using a one-sample $t$ test. A meaningful improvement would be 1 unit. With standard deviation of KDIS differences equaling 3 (a conservative assumption), sample sizes of 70 and 95 give $80 \%$ and $90 \%$ power based on a .05 level test.

\section{Dissemination plan}

The overall goal of our dissemination plan is to inform all stakeholders including patients, providers, payers, and government agencies about the process and findings of STUN Alcohol Use Now. The main message will be that small to medium-size primary care practices are partnering with NC AHEC to rapidly improve the implementation of SBI and MAT for unhealthy alcohol use. These efforts are expected to prevent deaths from unhealthy alcohol use as well as morbidity from the many adverse health consequences. We will commit to working with
AHRQ and its contractors in disseminating information about the project, such as through the AHRQ annual meeting and AHRQ publications. We will fully inform AHRQ and its designated contractors regarding implementation results and status of outcomes over time. All presentations and publications derived from STUN Alcohol Use Now will also be made available to AHRQ and its contractors.

By involving our stakeholders, we have already enlisted key people and organizations with the wherewithal and enthusiasm to support the dissemination of this project. We have begun to work closely with the NC AHEC Practice Support Program, the NCHQA, the NC Academy of Family Practice, Carolina Partners in Mental HealthCare, UNC's Alcohol and Substance Abuse Program, UNC's Virtual Care Center, and UNC-CH's practice-based research network, NCNet. AHEC has already informed their networks about the possibility of this work and the vast potential for reducing unhealthy alcohol use and improving outcomes using this proposal as a nidus. Additional specific organizations interested in research for unhealthy alcohol use that we intend to engage include the nascent accountable care organizations in $\mathrm{NC}$ and beyond, the $\mathrm{NC}$ Chapter of the American College of Physicians, the American Public Health Association, BCBS NC, the Society of General Internal Medicine, the National AHEC Organization, and Academy Health.

Once informed of the award, NC AHEC will be able to activate many associated practices before the actual funding using teams that already touch hundreds of primary care practices. The Steering Committee, that will include patient advocates yet to be named, will be regularly briefed on the process and results during every quarterly meeting. As we document evidence of process improvement, practice acceptance, and improved outcomes, we will provide the committee members with materials to distribute to their constituents without delay. The AHEC Practice Support Program remains closely connected to the NC Office of Health Benefits and Division of Public Health and often shares improvement results of ongoing efforts with these state agencies.

As we have done in the past, we will use community forums, local media outlets, scientific meetings, and publications to disseminate findings. AHEC is developing a presence on social media that could be used to disseminate unhealthy alcohol use awareness to new audiences. The practices involved in the actual study represent only a fraction of AHEC practices. The larger network provides a ripe environment for dissemination.

\section{Discussion}

This study will assess whether primary care practice facilitation can achieve dissemination and implementation 
of evidence-based screening and services for unhealthy alcohol use and will help clarify what practice support activities affect the uptake of recommended screening, brief interventions, and referral and treatment for unhealthy alcohol use. Additionally, this comprehensive evaluation will determine if there are differential practice outcomes based on the level of readiness for change and external factors in the practice environment.

Practice facilitators are trained "to meet the practice where they are" and adjust the approach to address barriers and reignite progress. Our experimental question is whether facilitation and other multi-modal supports can achieve fast initiation, spread, and improvement, especially since most practices have done little with systematic alcohol screening and treatment despite years of evidence supporting this work. We will set up the intervention process with the urgency of the task, understanding that the judgment of the facilitators may sometimes have to intervene and recalibrate the pace that a practice can maintain.

A strength of the NC AHEC practice support program is the ability of practice facilitators to assess what a practice needs to implement and to excel in process and outcome improvement $[62,63]$. We expect that practice facilitation and embedded telehealth may be effective because they each address key barriers. Barriers can include reticent providers and financial stressors. More subtle factors such as lack of trust, lack of knowledge, fear of change, and disbelief in the evidence can also pose obstacles to enacting organizational change. Specific to SBI for unhealthy alcohol use, competing priorities and limited time pose key barriers for primary care providers [19-21]. The initial screening itself can be done quickly, but we found that providers require an estimated 5-10 $\mathrm{min}$ to perform the screening-related assessment when a patient has positive screening results; additional time and visits are required for counseling those with risky drinking behavior [9, 19, 20, 64]. Other barriers specific to unhealthy alcohol use include that many providers/staff/practices lack knowledge of intervention options or training in delivery, or lack familiarity with the rationale and tools for implementation $[19,20]$. Further, it can be difficult to adapt EHRs to newer clinical measures. Finally, the small rural primary care practices in our study often have limited access to services (e.g., counseling for AUD) both within their practice and within the county.

Potential barriers to practice study enrollment include fear of financial risk, fully dedicating practice resources to other new activities such as Medicaid reform within the state or Accountable Care Organization participation, as well as perceptions of staffing issues, competing priorities, and lack of time. The mitigation strategy for these barriers will be to align practice facilitation to help the practice achieve some of these goals and offering incentives. Also, AHEC serves as a facilitator as it maintains strong relationships with most targeted practices marked by recurring meetings and continuing education programs. AHEC also maintains strong relationships with leading hospitals and health systems, and in some cases contracted work with many of the health systems involved in practice acquisitions. AHEC also supports primary care residency programs from which many participating providers graduated. We will leverage these relationships to boost accrual and prevent attrition. Instead of bringing in an intervention that is foreign to the practices, STUN will work with a known entity (NC AHEC) that can promote implementation through existing relationships, processes, and personnel.

If successful, our intervention would better identify and significantly reduce the burden of disease from unhealthy alcohol use in participating clinics. The study will produce fundamentally important evidence about the effect of practice facilitation on uptake of evidencebased SBI for unhealthy alcohol use when delivered on a large scale to small- to medium-sized primary care practices. It will also generate scientific knowledge about whether telehealth services can improve the use of evidence-based screening and interventions for practices with slower uptake. The results of this rigorously conducted evaluation are expected to have a positive impact by supplying evidence on effective and efficient dissemination and implementation of evidence related to addressing unhealthy alcohol use into primary care practices.

\section{Trial status}

At the time of manuscript completion, the trial is in the recruitment stage. Recruitment began in January 2020. This protocol is version 1, dated September 10, 2021. The end of the trial is expected in September 2023.

\section{Supplementary Information}

The online version contains supplementary material available at https://doi. org/10.1186/s13063-021-05641-7.

Additional file 1.

Additional file 2.

Additional file 3.

Acknowledgements

The authors gratefully acknowledge the essential contributions of the NC AHEC practice facilitators.

\section{Authors' contributions}

$D E J$ and $C B$ drafted the manuscript. DEJ led the development of the protocol, obtained the funding, and contributed to all aspects of the study design, recruitment plan, evaluation plan, and practice facilitation

intervention. $\mathrm{SAB}, \mathrm{DAD}, \mathrm{MHB}$, and $\mathrm{SC}$ contributed to the development of the evaluation framework and the evaluation plan. CB developed the

recruitment and intervention materials and provided material support. DG, 
$\mathrm{CW}, \mathrm{DAD}, \mathrm{MM}, \mathrm{SR}$, and $\mathrm{SC}$ contributed to the practice recruitment plan. $\mathrm{DG}$, $\mathrm{CW}, \mathrm{KAS}, \mathrm{MM}$, and SC contributed to the design of the practice facilitation component of the intervention and contributed to other aspects of the intervention and evaluation plan. MHB developed the statistical analysis plan. SR managed the data acquisition. The authors read, contributed to, and approved the final manuscript.

\section{Funding}

This research is supported by the Agency for Healthcare Research \& Quality (1R18HS027078-01), 5600 Fishers Lane, Rockville, MD 20857. Telephone: (301) 427-1364.

\section{Availability of data and materials}

The full study protocol is available through this publication and through the ClinicalTrials.gov record. The results of the study will be provided in ClinicalTrials.gov, when available, and through the publication of findings. The resources for primary care practices and materials used for the practice facilitation intervention are available on the study website (https:// stunalcoholusenow.org/).

\section{Declarations}

\section{Ethics approval and consent to participate}

All procedures performed in studies involving human participants were in accordance with the ethical standards of the University of North Carolina Institutional Review Board (UNC IRB) and with the 1964 Helsinki Declaration and its later amendments or comparable ethical standards. Informed consent was obtained electronically from all individual participants included in the study (i.e., the practice providers and staff who completed study surveys), via emails sent by study staff. The institutional review board approved a full waiver of informed consent for patients because patients at participating practices receive recommended routine care, and no individual-level patient data is collected by the study team. Important modifications to the protocol are submitted to the UNC IRB and to ClinicalTrials.gov.

\section{Consent for publication}

Not applicable

\section{Competing interests}

The authors declare that they have no competing interests.

\section{Author details}

'Division of General Internal Medicine and Geriatrics, Department of Internal Medicine, The Ohio State University, 2050 Kenny Road, Columbus, Ohio 43221, USA. ${ }^{2}$ Cecil G. Sheps Center for Health Services Research, CB 7590, University of North Carolina at Chapel Hill, Chapel Hill, NC 27599, USA. ${ }^{3}$ North Carolina Area Health Education Centers, CB 7165, University of North Carolina at Chapel Hill, Chapel Hill, NC 27599, USA. ${ }^{4}$ Department of Implementation Science, Wake Forest University School of Medicine, Winston-Salem, NC 27101, USA. ${ }^{5}$ Division of General Medicine and Clinical Epidemiology, CB 7110, University of North Carolina at Chapel Hill, Chapel Hill, NC 27599, USA. ${ }^{6}$ Department of Family Medicine, CB 7370, University of North Carolina at Chapel Hill, Chapel Hill, NC 27599, USA. 'Program on Health and Clinical Informatics, CB 7064, University of North Carolina at Chapel Hill, Chapel Hill, NC 27599, USA.

\section{Received: 26 March 2021 Accepted: 17 September 2021} Published online: 16 November 2021

\section{References}

1. Mokdad AH, Marks JS, Stroup DF, Gerberding JL. Actual causes of death in the United States, 2000. JAMA. 2004;291(10):1238-45. https://doi.org/10.1 001/jama.291.10.1238.

2. Centers for Disease Control and Prevention. BRFSS Prevalence \& Trends Data. 2013. Available at: http://nccd.cdc.gov/brfssprevalence/rdPage.a spx?rdReport=DPH_BRFSS.ExploreByLocation\&rdProcessAction=\&Sa veFileGenerated=1\&rdCSRFKey=503199fc-0542-4ed6-a807-c8b8106efa4 e\&is|Location=37\&is|Class=CLASS01\&isITopic=Topic03\&isIYear=2013\&hidLoca tion=37\&hidClass=CLASS01\&hidTopic=Topic03\&hidTopicName=Alcohol+ Consumption\&hidYear=2013\&irbShowFootnotes=Show\&icllndicators_rdExpa ndedCollapsedHistory=\&icllndicators=DRNKA
NY5\&hidPreviouslySelectedlndicators=\&DashboardColumnCount $=$ 2\&rdShowElementHistory=\&rdScrollX=0\&rdScrollY=320\&rdRnd=4248.

3. Centers for Disease Control and Prevention. Alcohol and Public Health: Centers for Disease Control and Prevention; 2015. Available at: http://www. cdc.gov/alcohol/data-stats.htm. Accessed 3 Sept 2021.

4. Woolf SH, Chapman DA, Buchanich JM, Bobby KJ, Zimmerman EB, Blackburn SM. Changes in midlife death rates across racial and ethnic groups in the United States: systematic analysis of vital statistics. BMJ. 2018; 362:k3096. https://doi.org/10.1136/bmj.k3096.

5. Woolf $\mathrm{SH}$, Schoomaker H. Life expectancy and mortality rates in the United States, 1959-2017. JAMA. 2019;322(20):1996-2016. https://doi.org/10.1001/ja ma.2019.16932.

6. Helping patients who drink too much: a clinician's guide. Rockville: National Institute on Alcohol Abuse and Alcoholism; Updated 2005 ed. Available at: http://pubs.niaaa.nih.gov/publications/Practitioner/CliniciansGuide2005/ guide.pdf. Accessed 3 Sept 2021.

7. Centers for Disease Control and Prevention. Alcohol Use and Your Health: Centers for Disease Control and Prevention; 2016. Available at: https://www cdc.gov/alcohol/fact-sheets/alcohol-use.htm. Accessed 3 Sept 2021.

8. Vinson DC, Manning BK, Galliher JM, Dickinson LM, Pace WD, Turner BJ. Alcohol and sleep problems in primary care patients: a report from the AAFP National Research Network. Ann Fam Med. 2010;8(6):484-92. https:// doi.org/10.1370/afm.1175.

9. Jonas DE, Garbutt JC, Brown JM, Amick HR, Brownley KA, Council CL, et al. Screening, behavioral counseling, and referral in primary care to reduce alcohol misuse. Rockville: Agency for Healthcare Research and Quality; 2012. Report No.: Comparative Effectiveness Review No. 64. (Prepared by the RTI International-University of North Carolina Evidence-based Practice Center under Contract No. 290200710056 I). AHRQ Publication No. 12-EHC055-EF.

10. O'Connor EA, Perdue LA, Senger CA, Rushkin M, Patnode CD, Bean SI, et al. Screening and behavioral counseling interventions to reduce unhealthy alcohol use in adolescents and adults: updated evidence report and systematic review for the US Preventive Services Task Force. JAMA. 2018; 320(18):1910-28. https://doi.org/10.1001/jama.2018.12086.

11. Curry SJ, Krist AH, Owens DK, Barry MJ, Caughey AB, Davidson KW, et al. Screening and behavioral counseling interventions to reduce unhealthy alcohol use in adolescents and adults: US Preventive Services Task Force Recommendation Statement. JAMA. 2018;320(18):1899-909. https://doi. org/10.1001/jama.2018.16789.

12. Miller WR, Rollnick SR. Motivational interviewing: preparing people to change behavior. New York: Guilford Press; 1991.

13. Rollnick S, Butler CC, Kinnersley P, Gregory J, Mash B. Motivational interviewing. BMJ. 2010;340(apr27 2):c1900. https://doi.org/10.1136/bmj.c1 900.

14. Jonas DE, Amick HR, Feltner C, Bobashev G, Thomas K, Wines R, et al. Pharmacotherapy for adults with alcohol use disorders in outpatient settings: a systematic review and meta-analysis. JAMA. 2014;311(18):1889900. https://doi.org/10.1001/jama.2014.3628.

15. D'Amico EJ, Paddock SM, Burnam A, Kung FY. Identification of and guidance for problem drinking by general medical providers: results from a national survey. Med Care. 2005;43(3):229-36. https://doi.org/10.1097/ 00005650-200503000-00005.

16. McKnight-Eily LR, Liu Y, Brewer RD, Kanny D, Lu H, Denny CH, et al. Vital signs: communication between health professionals and their patients about alcohol use -44 States and the District of Columbia, 2011. MMWR Morb Mortal Wkly Rep. 2014;63(01):16-22.

17. Fleming MF. Screening and brief intervention in primary care settings. Alcohol Res Health. 2004;28(2):57-62.

18. McNeely J, Kumar PC, Rieckmann T, Sedlander E, Farkas S, Chollak C, et al. Barriers and facilitators affecting the implementation of substance use screening in primary care clinics: a qualitative study of patients, providers, and staff. Addict Sci Clin Pract. 2018;13(1):8. https://doi.org/10.1186/s13722018-0110-8.

19. Barclay C, Viswanathan M, Jonas DE. Development of a primary care guide for implementing evidence-based screening and counseling for unhealthy alcohol use with epic-based electronic health record tools: a pilot dissemination project. Methods Research Report. (Prepared by RTI International-University of North Carolina Evidence-based Practice Center under Contract No. 290-2015-00011-I). Rockville: Agency for Healthcare Research and Quality; 2018. Report No.: AHRQ Publication No. 18-EHCO20-EF. 
20. Barclay C, Viswanathan M, Ratner SP, Tompkins J, Jonas DE. Implementing evidence-based screening and counseling for unhealthy alcohol use with epic-based electronic health record tools: a guide for clinics and health systems, developed as part of a pilot dissemination project (Prepared by the RTI International-University of North Carolina Evidence-based Practice Center under Contract No.290-2015-00011-1.). Rockville: Agency for Healthcare Research and Quality; 2018. Report No.: AHRQ Publication No. 18-EHC020-1-EF.

21. Jonas DE, Miller T, Ratner S, McGuirt B, Golin CE, Grodensky C, et al. Implementation and quality improvement of a screening and counseling program for unhealthy alcohol use in an academic general internal medicine practice. J Healthc Qual. 2017;39(1):15-27. https://doi.org/10.1097/ JHQ.0000000000000069.

22. Centers for Disease Control and Prevention. Ambulatory Care Use and Physician office visits: Centers for Disease Control; 2019. Available at: https:// www.cdc.gov/nchs/fastats/physician-visits.htm

23. Reinhart RJ. Nurses Continue to Rate Highest in Honesty, Ethics: Gallup. 2020. Available at: https://news.gallup.com/poll/274673/nurses-continue-ra te-highest-honesty-ethics.aspx.

24. Bauer MS, Damschroder L, Hagedorn H, Smith J, Kilbourne AM. An introduction to implementation science for the non-specialist. BMC Psychol. 2015;3(1):32. https://doi.org/10.1186/s40359-015-0089-9.

25. Langley GJ. The improvement guide: a practical approach to enhancing organizational performance. San Francisco: Jossey-Bass; 2009.

26. Baskerville NB, Liddy C, Hogg W. Systematic review and meta-analysis of practice facilitation within primary care settings. Ann Fam Med. 2012;10(1): 63-74. https://doi.org/10.1370/afm.1312.

27. Wang A, Pollack T, Kadziel LA, Ross SM, McHugh M, Jordan N, et al. Impact of practice facilitation in primary care on chronic disease care processes and outcomes: a systematic review. JGIM. 2018;33(11):1968-77. https://doi.org/1 0.1007/s11606-018-4581-9.

28. U.S. Department of Health \& Human Services. Available at: https://www.hhs. gov/about/news/2019/10/22/hhs-awards-16-million-help-primary-care-pra ctices-address-unhealthy-alcohol-use.html. Accessed 3 Sept 2021.

29. Tippett R. North Carolina's Hispanic Community: 2019 Snapshot: Carolina Demography. 2019. Available at: https://www.ncdemography.org/2019/09/2 6/north-carolinas-hispanic-community-2019-snapshot/\#: :text=Statewide\%2 C\%209.6\%25\%20of\%20North\%20Carolina's,the\%20national\%20average\%20(1 8.3\%25)

30. North Carolina Department of Health and Human Services. North Carolina Alcohol Data Dashboard. Available at: https://public.tableau.com/profile/nc. injury.and.violence.prevention.branch\#!/vizhome/NCAlcoholDataDashboard/ Story. Accessed 3 Sept 2021.

31. America's Health Rankings. Explore excessive drinking in North Carolina: 2018 Annual Report. Available at: https://www.americashealthrankings.org/ explore/annual/measure/ExcessDrink/state/NC. Accessed 3 Sept 2021.

32. Stahre M, Roeber J, Kanny D, Brewer RD, Zhang X. Contribution of excessive alcohol consumption to deaths and years of potential life lost in the United States. Prev Chronic Dis. 2014;11:E109. https://doi.org/10. 5888/pcd11.130293.

33. Witt AA, Berkowitz SA, Gillberg C, Lowe MR, Råstam M, Wentz E. Weight suppression and body mass index interact to predict long-term weight outcomes in adolescent-onset anorexia nervosa. J Consult Clin Psychol. 2014;82(6):1207-11. https://doi.org/10.1037/a0037484.

34. Program on Health Workforce Research and Policy. North Carolina Health Professional Supply Data. Available at: https://nchealthworkforce.unc.edu/ interactive/supply/. Accessed 3 Sept 2021.

35. Chan AW, Tetzlaff JM, Gøtzsche PC, Altman DG, Mann H, Berlin JA, et al. SPIRIT 2013 explanation and elaboration: guidance for protocols of clinical trials. BMJ. 2013;346(jan08 15):e7586. https://doi.org/10.1136/bmj.e7586.

36. Rodriguez LM, Litt DM, Stewart SH. Drinking to cope with the pandemic: the unique associations of COVID-19-related perceived threat and psychological distress to drinking behaviors in American men and women. Addict Behav. 2020;110:106532. https://doi.org/10.1016/j.addbeh.2020.1 06532.

37. Wardell JD, Kempe T, Rapinda KK, Single A, Bilevicius E, Frohlich JR, et al. Drinking to cope during COVID-19 pandemic: the role of external and internal factors in coping motive pathways to alcohol use, solitary drinking, and alcohol problems. Alcohol Clin Exp Res. 2020;44(11):2150-3.

38. National Institute on Alcohol Abuse and Alcoholism. Surveillance report COVID-19: alcohol sales during the COVID-19 pandemic. Available at:
https://pubs.niaaa.nih.gov/publications/surveillance-covid-19/COVSALES.htm. Accessed 3 Sept 2021.

39. Practice support: what we do: North Carolina AHEC; 2020. Available at: https://www.ncahec.net/. Accessed 3 Sept 2021.

40. Science of improvement: testing changes institute for healthcare improvement; 2018. Available at: http://www.ihi.org/resources/Pages/ Howtolmprove/ScienceoflmprovementHowtolmprove.aspx. Accessed 3 Sept 2021.

41. Wagner EH, Austin BT, Davis C, Hindmarsh M, Schaefer J, Bonomi A. Improving chronic illness care: translating evidence into action. Health Aff (Project Hope). 2001;20(6):64-78. https://doi.org/10.1377/hlthaff.20.6.64.

42. Coleman K, Austin BT, Brach C, Wagner EH. Evidence on the Chronic Care Model in the new millennium. Health Aff (Project Hope). 2009;28(1):75-85. https://doi.org/10.1377/hlthaff.28.1.75.

43. Institute for Healthcare Improvement. The breakthrough series: IHI's collaborative model for achieving breakthrough improvement. Diabetes Spectr. 2004;17(2):97.

44. Bandura A. Social learning theory. Englewood Cliffs: Prentice-Hall; 1977.

45. Speck M. Best practice in professional development for sustained educational change. ERS Spectr. 1996;14(2):33-41.

46. Solberg LI, Asche SE, Margolis KL, Whitebird RR. Measuring an organization's ability to manage change: the change process capability questionnaire and its use for improving depression care. Am J Med Qual. 2008;23(3):193-200. https://doi.org/10.1177/1062860608314942.

47. Jaen CR, Crabtree BF, Palmer RF, Ferrer RL, Nutting PA, Miller WL, et al. Methods for evaluating practice change toward a patient-centered medical home. Ann Fam Med. 2010;8(Suppl 1):S9-20; s92.

48. Nutting PA, Crabtree BF, Stewart EE, Miller WL, Palmer RF, Stange KC, et al Effect of facilitation on practice outcomes in the National Demonstration Project model of the patient-centered medical home. Ann Fam Med. 2010; 8(Suppl 1):S33-44; s92.

49. Scholle SH, Asche SE, Morton S, Solberg LI, Tirodkar MA, Jaen CR. Support and strategies for change among small patient-centered medical home practices. Ann Fam Med. 2013;11(Suppl 1):S6-13. https://doi.org/10.1370/a fm. 1487.

50. Shea CM, Jacobs SR, Esserman DA, Bruce K, Weiner BJ. Organizational readiness for implementing change: a psychometric assessment of a new measure. Implement Sci. 2014;9(1):7. https://doi.org/10.1186/1748-5908-9-7.

51. Weiner BJ. A theory of organizational readiness for change. Implement Sci. 2009;4(1):67. https://doi.org/10.1186/1748-5908-4-67.

52. Weiner BJ, Amick H, Lee SY. Conceptualization and measurement of organizational readiness for change: a review of the literature in health services research and other fields. Med Care Res Rev. 2008;65(4):379-436. https://doi.org/10.1177/1077558708317802.

53. Klein KJ, Sorra JS. The challenge of innovation implementation. Acad Manag Rev. 1996;21(4):1055-80. https://doi.org/10.5465/amr.1996.9704071863.

54. Weiner BJ, Lewis MA, Linnan LA. Using organization theory to understand the determinants of effective implementation of worksite health promotion programs. Health Educ Res. 2009;24(2):292-305. https://doi.org/10.1093/her/ cyn019.

55. Helfrich CD, Weiner BJ, McKinney MM, Minasian L. Determinants of implementation effectiveness: adapting a framework for complex innovations. Med Care Res Rev. 2007;64(3):279-303. https://doi.org/10.11 77/1077558707299887.

56. Agency for Healthcare Quality and Research. Development of the Clinician \& Group Surveys 2013. Available at: https://cahps.ahrq.gov/surveys-guida nce/cg/about/Develop-CG-Surveys.html. Accessed 3 Sept 2021.

57. Teal R, Bergmire DM, Johnston M, Weiner BJ. Implementing communitybased provider participation in research: an empirical study. Implement Sci. 2012;7(1):41. https://doi.org/10.1186/1748-5908-7-41.

58. CHIRr: Consumer Health Informatics Research Resource. Technology Acceptance Model (Perceived Usefulness and Perceived Ease of Use). Bethesda: National Institutes of Health. Available at: https://chirr.nlm.nih.gov/ tam.php. Accessed 3 Sept 2021.

59. Davis FD. Perceived usefulness, perceived ease of use, and user acceptance of informaion technology. MIS Q. 1989;13(3):319-40. https://doi.org/10.23 07/249008.

60. Donner A, Klar N. Pitfalls of and controversies in cluster randomization trials. Am J Public Health. 2004;94(3):416-22. https://doi.org/10.2105/AJPH.94.3.416.

61. Esserman D, Allore HG, Travison TG. The method of randomization for cluster-randomized trials: challenges of including patients with multiple 
chronic conditions. Int J Stat Med Res. 2016;5(1):2-7. https://doi.org/10. 6000/1929-6029.2016.05.01.1.

62. Cykert S, Keyserling TC, Pignone M, DeWalt D, Weiner BJ, Trogdon JG, et al. A controlled trial of dissemination and implementation of a cardiovascular risk reduction strategy in small primary care practices. Health Serv Res. 2020; 55(6):944-53.

63. Cykert S, Lefebvre A, Bacon T, Newton W. Meaningful use in chronic care: improved diabetes outcomes using a primary care extension center model. NC Med J. 2016;77(6):378-83. https://doi.org/10.18043/ncm.77.6.378.

64. Jonas DE, Garbutt JC, Amick HR, Brown JM, Brownley KA, Council CL, et al. Behavioral counseling after screening for alcohol misuse in primary care: a systematic review and meta-analysis for the U.S. Preventive Services Task Force. Ann Intern Med. 2012;157(9):645-54. https://doi.org/10.7326/0003-481 9-157-9-201211060-00544.

\section{Publisher's Note}

Springer Nature remains neutral with regard to jurisdictional claims in published maps and institutional affiliations.

Ready to submit your research? Choose BMC and benefit from:

- fast, convenient online submission

- thorough peer review by experienced researchers in your field

- rapid publication on acceptance

- support for research data, including large and complex data types

- gold Open Access which fosters wider collaboration and increased citations

- maximum visibility for your research: over $100 \mathrm{M}$ website views per year

At $\mathrm{BMC}$, research is always in progress.

Learn more biomedcentral.com/submissions 